\title{
IL CORPUS RHYTHMORUM MUSICUM OPPORTUNITÀ E PROSPETTIVE DI UNA EDIZIONE CRITICA DIGITALE
}

\author{
Irene Volpi \\ Società Internazionale per lo Studio del Medioevo Latino \\ volpirene@gmail.com
}

\section{Edizioni critiche e filologia digitale}

Negli ultimi anni, si potrebbe dire negli ultimi mesi, sono nati e continuano a essere implementati molti siti per la ricerca nel campo dei manoscritti e delle melodie. Lavori ormai imprescindibili, non tanto per la facilità del loro reperimento rispetto alle versioni cartacee, quanto piuttosto per il continuo aggiornamento e per la possibilità di incrociare i dati ivi contenuti, che permettono di avere in breve tempo un quadro della situazione documentaria, quando prima sarebbero occorsi molti viaggi e molto tempo da dedicarvi. In particolare - ne citiamo solamente alcuni relativi all'alto medioevo e le esclusioni, necessarie, non vogliono indicare una minore affidabilità o interesse - ci sembrano degne di nota le iniziative che riescono a connettere diversi tipi di informazioni e, prima ancora, di competenze degli studiosi che le hanno pensate e attuate. Penso a Cantus Index ${ }^{1}$, repertorio dei testi cantati e delle melodie utilizzate nella Messa e nell'Officio, che, grazie a un unico Cantus ID number condiviso, ha potuto integrare altri progetti, quali Cantus Manuscripts Database, «[a] searchable digital archive holds inventories of primarily antiphoners and breviaries from medieval Europe» ${ }^{2}-$ che riprende

\footnotetext{
${ }^{1}$ «Cantus Index. Online catalogue for Mass and Office chants», a cura di D. Lacoste. Link: $<$ http:// cantusindex.org/> [consultato in data: 06/11/2019].

${ }^{2}$ «Cantus Manuscript Database», a cura di D. Lacoste, Canada, University of Waterloo, 2012. Link: $<$ http://cantus.uwaterloo.ca/ $>$ [consultato in data: 06/11/2019]. I curatori fanno inoltre sapere che «New
} 
e aggiorna i dati contenuti nei Monumenta Monodica Medii Aevi (MMMA) ${ }^{3}$ - e $M M M O$ Database $e^{4}$, aggiunto di recente, per la raccolta delle riproduzioni digitali dei manoscritti con notazione. Anche Cantus Planus dell'Università di Regensburg ${ }^{5}$, che ospita vari datafiles e databases sul canto gregoriano, permette ricerche combinate sui canti dell'Officio studiati nei voll. 3 e 4 del Corpus Antiphonalium Officii $(\mathrm{CAO})^{6}$, nonché di consultare le scansioni dei Monumenti vaticani di paleografia musicale latina di Bannister ${ }^{7}$.

Il database del Corpus Rhythmorum Musicum (saec. IV-IX) I. Songs from non-liturgical sources $(\mathrm{CRM})^{8}$ parte dal medesimo materiale, poesia e musica, ma ha una diversa finalità, poiché qui, sfruttando pienamente le opportunità rese disponibili dal supporto informatico, si propone l'edizione critica dei testi, assieme alle trascrizioni e alle immagini di tutti i manoscritti, così da permettere al ricercatore un controllo diretto, sia sulle scelte operate dal curatore, sia sulle fonti stesse. L'edizione, in tal modo, non oscura le lezioni scartate né censura i manoscritti che possono riportare diverse redazioni; questi si rivelano testimoni preziosi di determinati ambienti culturali che lessero e ascoltarono l'opera come ci è stata tramandata da quel codice e non nella versione ricostruita. Parimenti, non si rinuncia a indagare i rapporti tra i testi tramandati per cercare di comprendere gli intenti e lo stile dell'autore, proponendo lo stemma codicum e l'edizione, ma anche per conoscere la storia successiva dell'opera e delle sue modalità di fruizione, poiché sia la cultura, l'ambiente e le scelte grammaticali e stilistiche dell'autore, sia le innovazioni e i cambiamenti linguistici introdotti dai copisti ci sembrano degni di essere segnalati e analizzati. Nell'edizione a stampa, problemi di carattere tecnico e di spazio non permettono simili raffronti e impongono la selezione dei dati da rendere disponibili; per uno studio comparativo sulla fortuna e i lasciti

phases of the project include adding chant melodies to existing records and indexing manuscripts for the Mass, including those sources that contain sequences».

${ }^{3}$ Bruno Stäblein, «Hymnen, Die mittelalterlichen Hymnenmelodien des Abendlandes», in Monumenta Monodica Medii Aevi, vol. 1, Kassel-Bassel, 1995.

${ }^{4}$ «Medieval Music Manuscripts Online Database», a cura di D. Gatté, ultima revisione del 8/21/2018. [On line]. Link: $<\mathrm{http}: / /$ musmed.eu/> [consultato in data: 21/08/2019].

${ }^{5}$ «Cantus planus», a cura di D. Hiley e R. Klugseder, Regensburg, 2014. Link: $<$ https://www.uni-regensburg.de/Fakultaeten/phil_Fak_I/Musikwissenschaft/cantus/ $>$ [consultato in data: 06/11/2019].

${ }^{6}$ Renato-Joanne Hesbert, Corpus Antiphonalium Officii, vol. III, Invitatoria et Antiphonae, vol. IV, Responsoria, Versus, Hymni et varia, Roma, Casa editrice Herder, 1968 e 1970.

${ }^{7}$ Henri Marriot Bannister, Monumenti vaticani di paleografia musicale latina, vol. 12 di Codices e vaticanis selecti, phototypice expressi, Leipzig, Otto Harrassowitz, 1913, reprint Farnborough, Gregg, 1969.

${ }^{8}$ Corpus Rhythmorum Musicum (saec. IV-IX) I. Songs from non-liturgical sources - Canti di tradizione non liturgica, a cura di F. Stella, Millennio Medievale Testi, Firenze, SISMEL, Edizioni Del Galluzzo, 2007; edizione digitale coordinata da F. Stella, edizione musicale a cura di S. Barrett. Link: <http://www. corimu.unisi.it> [consultato in data: 06/11/2019]. 
di un'opera, queste costrizioni e le inevitabili esclusioni, anche in apparato, possono essere deleterie, perché rischiano di falsificare la realtà storica e di condurre il ricercatore a conclusioni errate: «non è affatto vero che in filologia i danni non sono irreversibili per il solo fatto che le fonti primarie, cioè i manoscritti, continuano a essere consultabili. Quello che circola sono le edizioni, non i manoscritti, e nella medievistica è estremamente raro che di un testo si faccia più di un'edizione per ogni secolo»? .

Le edizioni tradizionali, ma anche quelle della cosiddetta New philology basate su un codex unicus $^{10}$, propongono al lettore solamente uno stadio della tradizione, più o meno risalente al testo voluto dall'autore, e dunque accentuano il divario tra testo ricostruito e quello testimoniato dal singolo manoscritto. Con questo tipo di edizione digitale si vuole tentare di colmare tale distanza, proponendo una ricostruzione che sia quanto più possibile trasparente e verificabile, dimodoché anche l'eventuale errore possa essere presto discusso e corretto, grazie al dialogo con tutta la comunità scientifica:

Beyond the editing, the scientific objectives are to reconstruct the original performative nature of these compositions, and to systematically and statistically study the birth of this versification and moreover to use rhythmical poems as documentary evidence of the linguistic transition from Latin to early Romance. [...] This process overcomes the distinction between a Text reconstructed by the editor and the many transmitted texts, which are usually shattered, misrepresented, and left unrecognizable in the apparatus of the variants ${ }^{11}$.

\footnotetext{
${ }^{9}$ Francesco Stella, «Metodi e prospettive dell'edizione digitale di testi mediolatini». Link: $<$ https:// diazilla.com/doc/1001195/metodi-e-prospettive-dell-edizione-digitale-di-testi-medi... [consultato in data: 06/11/2019].

${ }^{10}$ «In generale, si ha l'impressione che i risultati più importanti, quelli in cui la differenza specifica dell'edizione digitale è particolarmente vistosa, siano stati raggiunti in casi dove il rapporto fra i manoscritti e i testi editi è di uno a uno» (ibidem); essi hanno permesso di applicare le tecniche di marcatura e di raggiungere una sofisticata visualizzazione di dati multipli, inimmaginabile su supporto cartaceo, nel campo, ad esempio, della filologia germanica o romanza. Più povero, da questo punto di vista, è l'ambito mediolatino, il quale, più che di edizioni digitali, può semmai avvalersi del database «Mirabile, Archivio digitale per la cultura medievale». Link: $<$ http://mirabileweb.it/> [consultato in data: 06/11/2019]. Promosso dalla Società per lo Studio del Medioevo Latino (SISMEL) e dalla Fondazione Ezio Franceschi$n i$ (FEF), che include, per la parte mediolatina, i seguenti progetti: MEL (MEdioevo Latino, Bollettino bibliografico della cultura europea da Boezio a Erasmo, sec. VI-Xv); BISLAM (BIbliotheca Scriptorum LAtinorum Medii Recentiorisque Aevi); CALMA (Compendium Auctorum Latinorum Medii Aevi); MEM (MEdioevo Musicale, Bollettino bibliografico della musica medievale); RICaBIM (Repertorio di Inventari e CAtaloghi delle BIblioteche Medievali di area latina dall'Alto Medioevo sino al 1520); Canticum (repertorio dei codici che tramandano commenti al Cantico dei Cantici); ABC (Antica Biblioteca Camaldolese); ROME (Repertorio degli Omeliari del MEdioevo); TETRA (trasmissione dei testi latini del Medioevo); TRAMP (TRAdizione Medievale dei Padri), MADOC (MAnuscripta DOCtrinalia, sec. XIII-Xv).

${ }^{11}$ Francesco Stella, Formes strophiques simples - Simple strophic patterns, a cura di L. Seláf, P. Noel, A. Hanna, J. van Driel, Budapest, Akadémiai Kiadó, 2010, p. 20.
} 


\section{Poesia e musica nei rhithmi}

Un altro tema cruciale è il legame vivo e complesso tra testo, melodia e testimonianza manoscritta: ne risultano le edizioni critiche, oltre che delle poesie, delle trascrizioni neumatiche, nonché una gran quantità di dati e di tabelle gratuitamente disponibili agli studiosi. Con questo progetto, inoltre, si vuole portare un contributo alla critica, confrontarsi su ciò che è considerato poesia, riflettervi da rinnovate, ma antichissime, prospettive.

I secoli presi in esame, che culminano con la rinascita carolingia, sono la fucina che, selezionando materiali vecchi e nuovi, li fonde in nuove unità, dalle quali prenderà le mosse tutta la cultura occidentale, sia in lingua latina, sia in quelle volgari. Tra questi elementi strutturali, il legame intrinseco di molte di tali forme poetiche con la musica rimane quello meno indagato dagli specialisti, benché studiosi come Avalle prima, e ora Lannutti per la poesia romanza, abbiano richiamato l'attenzione su questo nesso e ne abbiano studiato le conseguenze nella forma ${ }^{12}$ e nella tradizione manoscritta ${ }^{13}$. Se pure la melodia può rivelarsi precedente o successiva al testo, essa non rimane, per quei poeti, elemento esornativo, ma strutturante; ancora Dante, seppur ormai lontano dalle prime poesie volgari nate in simbiosi con la musica, lo ricorda con insistenza nel De Vulgari Eloquentia, sia a proposito della conformazione della strofa, sia, più direttamente, nella presentazione del rapporto tra musica e testo poetico:

Nullus enim tibicen, vel organista, vel cytharedus melodiam suam cantionem vocat, nisi in quantum nupta est alicui cantioni; sed armonizantes verba opera sua cantiones vocant, et etiam talia verba in cartulis absque prolatore iacientia cantiones vocamus. Et ideo cantio nihil aliud esse videtur quam actio completa dictantis verba modulationi armonizata ${ }^{14}$.

${ }^{12}$ Silvio d'Arco Avalle, Le forme del canto. La poesia nella scuola tardoantica e altomedievale, a cura di M. S. Lannutti, Firenze, Edizioni del Galluzzo per la Fondazione Ezio Franceschini, 2017; tra i tanti articoli di M. Sofia Lannutti, citiamo «Anisosillabismo e semiografia musicale nel laudario di Cortona», in Studi medievali, 35 (1994), pp. 1-66.

${ }^{13}$ Cfr., ad esempio, M. Sofia Lannutti, «Musica e irregolarità di versificazione nella tradizione dei testi lirici latini e romanzi», in Filologia mediolatina, 15 (2008), pp. 115-131; «Versificazione francese irregolare tra testo verbale e testo musicale», in Studi di filologia medievale offerti a d'Arco Silvio Avalle, Milano-Napoli, Ricciardi, pp. 185-215.

${ }^{14}$ «Nessun suonatore di strumenti a fiato o a testiera o a corde definisce come "canzone" la sua melodia, se non in quanto è accoppiata a qualche canzone, mentre chi dispone parole secondo le leggi dell'armonia chiama le sue opere "canzoni", e quelle parole sono chiamate "canzoni" anche quando se ne stanno scritte su qualche foglio senza che nessuno le reciti. La canzone non è quindi altro che l'azione compiutamente svolta di chi dispone parole armonizzandole in vista della modulazione melodica». Dante 
$\mathrm{Al}$ di là delle discussioni ancora aperte sulla frequenza con cui le canzoni italiane siano state effettivamente cantate tra Due e Trecento (nonché sulla distanza formale di queste dai rhythmi mediolatini), la definizione dell'Alighieri rimane preziosa perché testimonia una forma mentis che viene da lontano, in un periodo per il quale già si parla, a torto o a ragione, di «divorzio tra poesia e musica» in Italia.

La volontà di creare un componimento con (o per il quale era comunque prevista) la melodia, infatti, fa vertere l'autore verso determinate scelte stilistiche, solitamente evitate nella poesia latina classica e per questo motivo a lungo considerate dalla critica, con un certo pregiudizio, deteriori, sintomo di decadenza culturale e della incapacità di innovazione.

Questo vale per molti espedienti poetici utilizzati nell'alto medioevo, quali: la paratassi, talora al limite dell'anacoluto o strutturata in una lunga serie di ossimori e parallelismi; la versificazione non rigorosa, sia nel conteggio delle sillabe, sia nella cadenza finale o, eventualmente, a fine emistichio - si confronti a tal proposito le tabelle metriche del Corpus, in particolare le sezioni dedicate a ipo/ipermetrie, diastole e sistole -; la presenza di formule ripetitive e fisse (si veda, quale esempio, la rassegna di elementi ricorrenti nei rhythmi legati all'esegesi e agli episodi della Bibbia in La poesia latina carolingia a tema biblico $^{15}$ ). Eppure, tali espedienti non assumono necessariamente un valore negativo, specie se teniamo conto che questi coabitavano con altri elementi architettonici, quali, in primis, la melodia, le omofonie (si pensi allo sviluppo della rima nell'innologia e nei rhythmi mediolatini, all'allitterazione in ambito irlandese), la formula abecedaria, adottata già da Agostino nel suo Psalmus contra partem Donati, nonché l'introduzione fortunatissima del ritornello. In molti casi la scelta di questi elementi strutturanti non era né casuale, né l'unica attuabile, come dimostrano le composizioni ritmiche di autori che pure erano in grado di comporre versi perfettamente metrici, quali Agostino, Beda, Alcuino, Paolino d'Aquileia e Rabano Mauro, bensì mirava alla memorabilità e alla divulgazione essenzialmente orale del testo, più che alla conservazione dello stesso. L'integrità testuale può essere preservata dagli autori con altre tipologie di meccanismi ${ }^{16}$, quali gli intrecci

Alighieri, De vulgari eloquentia, a cura di E. Fenzi, con la collaborazione di L. Formisano e F. Montuori, Roma-Salerno, Nuova edizione commentata delle opere di Dante, 3, 2012, p. 203.

${ }^{15}$ Francesco Stella, op. cit. Anche negli inni ritroviamo, amplificato, il medesimo procedimento, così, più tardi, nelle sequenze, prima latine e poi volgari.

${ }^{16}$ Rispetto a queste problematiche, si può consultare, per la poesia italiana sino a Petrarca, il volume di Maria Clotilde Camboni, Fine Musica. Percezione e concezione delle forme della poesia. Dai siciliani a Petrarca, Fellowship Marco Praloran 2, Quaderni di Stilistica e Metrica Italiana 8, Firenze, Edizioni del Galluzzo, 2017. 
di rime complessi e articolati, variazioni non banali di metri all'interno della strofa, richiami continui e non occasionali tra le strofe: espedienti ai quali, non a caso, non si fa ricorso nei rhythmi, dove l'intento divulgativo, mnemonico e didattico è preponderante rispetto a quello della rimarcata autorialità e della raffinatezza stilistica.

\section{Il Corpus Rhythmorum Musicum}

Il CRM è dedicato all'edizione critica di queste poesie latine musicate e ritmiche - fondate cioè sul ritmo determinato dalla successione degli accenti in versi di un numero di sillabe definito (principio sillabotonico) - dei secoli IV-IX. Il progetto, ideato da Francesco Stella e promosso dall'Università di Siena e dalla Società Internazionale per lo Studio del Medioevo Latino (SISMEL), ha portato nel dicembre 2007 alla pubblicazione del primo volume con CR-ROM sui Canti di tradizione non liturgica, cui è seguita la versione online. Il Corpus dà edizione critica di tali testi, corredandoli con la trascrizione delle notazioni musicali curata da Sam Barrett, con le registrazioni delle esecuzioni canore (dirette da Giacomo Baroffio), le immagini dei manoscritti, l'apparato filologico, gli indici statistici relativi a questioni linguistiche, le tabelle metriche e le tavole neumatiche, offrendo così una edizione "aperta", con una grande mole di materiale comparabile su cui condurre diversi tipi di ricerca. A questo scopo è stato sviluppato da Luigi Tessarolo un apposito database che ha permesso di rappresentare tutti gli aspetti filologici, metrici, linguistici e musicali dei testi inseritivi:

A questo proposito confidiamo sull'utilità che, per una considerazione sistematica dei problemi, potrà avere una riedizione dei testi che tenga maggior conto delle molteplicità di redazioni e del contesto performativo, musicale, e in ultima analisi orale attestato dalla tradizione manoscritta, e la disponibilità completa di materiale critico e di dati filologici confrontabili grazie a un motore di ricerca ${ }^{17}$.

Al fine di facilitare questi confronti, nel CRM, oltre alla consultazione delle singole schede, è possibile visualizzare i dati incrociati nelle tabelle metriche, che contengono un elenco, curato da E. D'Angelo, di tutte le forme di versi ritmici attestate nella poesia mediolatina, e in quelle linguistiche. Inoltre, è possibile porre al database domande complesse e particolareggiate

${ }^{17}$ Francesco Stella, «Introduzione all'edizione critica», in Corpus Rhythmorum Musicum, op. cit. 
nel Menù di navigazione Ricerca, dove l'interrogazione può riguardare sia il testo in generale, sia dati linguistici e metrici, oltre che musicali.

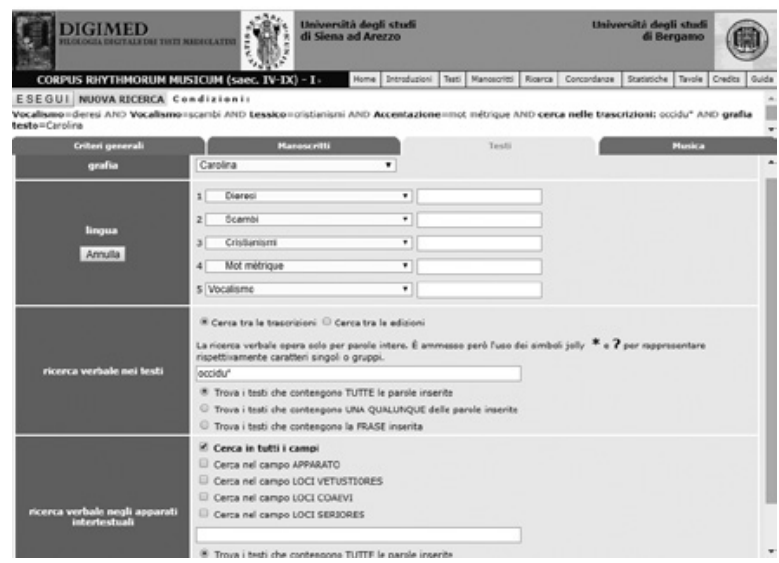

FIGURA 1. Finestra di ricerca, particolare sui dati testuali e linguistici.

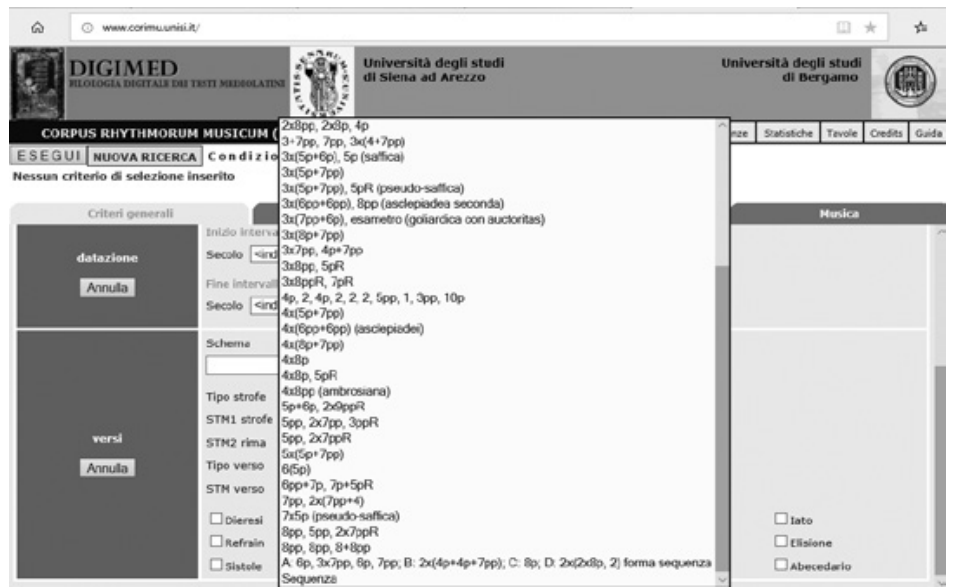

FIgURA 2. Finestra di ricerca; particolare sui dati metrici, con aperto l'elenco delle strofe selezionabili.

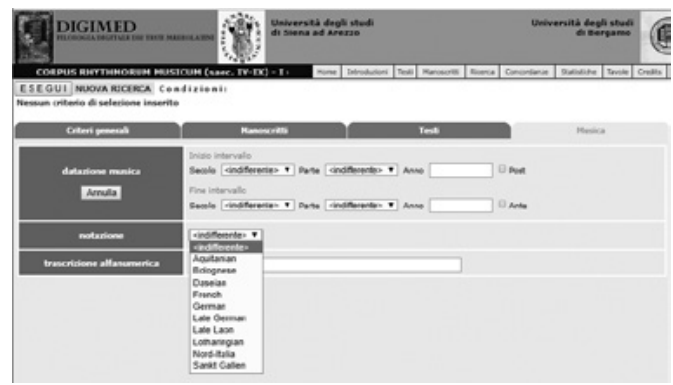

FIGURA 3. Finestra di ricerca, sezione musicale. 
Questo tipo di ricerca, grazie alla trascrizione alfanumerica codificata da Sam Barrett, dà la possibilità di interrogare il database su una specifica porzione di testo musicale, permettendo così delle comparazioni non soltanto all'interno del CRM, ma anche con altri brani, in modo da indagare i casi di contraffazione di una poesia sulla base di una melodia precedente e viceversa, oppure di studiare il rapporto di brani musicali, toni, intonazioni con determinati generi o strofe.

La riproduzione di tutti i manoscritti, compresi ovviamente quelli con neumi, assieme alle esecuzioni e alle trascrizioni musicali, consente l'indagine del rapporto tra singolo testo e melodia, tra le anomalie versificatorie e la trascrizione neumatica: talvolta un manoscritto può contenere un testo con versi anisosillabici dovuti a errori del copista, e chi correda questo brano di neumi, li adegua con varie strategie a queste anomalie. Si può dare anche il caso contrario: di fronte a un testo corrotto, il copista può aver emendato i versi anisosillabici in base alla melodia nota; talora, infine, se la musica conosciuta dal copista è leggermente diversa da quella prevista per il testo da trascrivere, lo scriba può comunque decidere di intervenire sulla poesia piuttosto che sui neumi. Una interessante casistica, corredata di esempi, si trova nell'articolo Ad cantandum carmina ${ }^{18}$.

Per facilitare l'uniformità nella raccolta delle informazioni (e dunque la coerenza delle statistiche), sono state seguite alcune linee guida per l'edizione dei testi nel CRM:

- rispetto del testo tradito per evitare alterazioni dei dati riguardanti la versificazione e la lingua, permettendo così un'analisi e un'attenzione maggiore alla genesi dei fenomeni anisosillabici;

- trascrizione in apparato anche delle varianti grafiche, «preziose per le ricostruzioni linguistiche e per la collocazione geografica del testo» ${ }^{19}$;

- la proposta, ove possibile, di uno stemma codicum.

Oltre alla constitutio textus con apparato, per ciascun ritmo si eseguono le ricerche elencate di seguito:

- Il confronto con la Bibbia e le altre composizioni, anche liturgiche e paraliturgiche, con esplicitazione dei loci vetustiores, coaevi, seriores, per la costruzione dell'apparato intertestuale. Questo lavoro mira a fornire dati utili alla storia di singole espressioni e all'individuazione di correlazioni fra testi poetici che hanno permesso talora di fornire «indicazioni di grande

${ }^{18}$ Francesco Stella, «Ad cantandum carmina», op. cit., pp. 333-353.

${ }^{19}$ Francesco Stella, «Introduzione all'edizione critica», in Corpus Rhythmorum Musicum, op. cit. 
peso per la datazione o l'attribuzione del ritmo, e che in tutti i casi aiutano a ricostruire l'ambiente culturale di produzione e di fruizione» ${ }^{20}$.

- L'analisi accurata della versificazione (la rilevanza della stessa è amplificata dalla diffusione e dalla frequenza con cui tali composizioni erano cantate), volta a comprendere la storia e la fortuna di determinate forme ritmiche mediolatine, oltre alla predominante quartina di dimetri ambrosiani.

- L'analisi linguistica che espliciti sia «le divergenze più evidenti del testo rispetto alla grammatica convenzionale del latino classico e postclassico», sia «la distanza o [...] la prossimità del latino di queste poesie agli sviluppi romanzi: statuto sociolinguistico (livello culturale dell'autore, destinatari, zona linguistica $)^{21} \gg$.

- L'edizione del testo musicale ${ }^{22}$ e la messa in luce dei legami testo-musica, sia a livello generale ${ }^{23}$ che particolare, dove il rapporto con il testo musicale andrà considerato caso per caso, data la menzionata possibilità dell'esistenza di più melodie per un solo testo poetico, ma anche il caso contrario, secondo tradizioni perlopiù locali.

Il tratto caratteristico del Corpus rimane il legame di queste antiche composizioni con la musica: esso permette di avere, in uno stesso contenitore, l'edizione critica del testo e quella musicale, e prevede una descrizione sia della melodia in generale, sia della singola trascrizione neumatica in rapporto con il testo contenuto nello stesso manoscritto.

\section{${ }^{20}$ Ibidem.}

${ }^{21}$ Ibidem.

${ }^{22}$ Cfr. Sam Barrett, «Ritmi ad cantandum: some preliminary editorial consideration», in Poesia dell'Alto Medioevo europeo: manoscritti, lingua e musica dei ritmi latini, a cura di F. Stella, prefazione di C. Leonardi, atti delle euroconferenze per il Corpus dei ritmi latini (4.-9. sec.) (Arezzo 6-7 novembre 1998 e Ravello 9-12 settembre 1999), Firenze, SISMEL, Edizioni del Galluzzo, 2000; «On editing neumatic notations on rhythmical verse», in Poetry of the Early Medieval Europe: Manuscripts, Language and Music of the Rhythmical Latin Texts, a cura di E. D'Angelo e F. Stella, prefazione di B. K. Vollmann, III Euroconference for the digital edition of the Corpus of Latin Rhythmical Texts $4^{\text {th }}-9^{\text {th }}$ Century (Munich, 2-4 november 2000), Firenze, SISMEL, Edizioni del Galluzzo, 2003, pp. 149-169.

${ }^{23}$ Come, ad esempio, già rilevava Norberg per la forma strofica del dimetro ambrosiano $(8 \mathrm{pp}, 8 \mathrm{pp}$, $8+8 \mathrm{pp}$, secondo il sistema di rappresentazione dei versi ritmici da lui elaborato), in cui la costruzione è determinata dall'architettura melodica AABA. Cfr. Dag Norberg, An introduction to the study of medieval Latin versification, traduzione di G. C. Roti, a cura di J. Ziolkowski, Washington D.C., The Catholic University of America Press, 2004, p. 133: «We could emphasize this fact by giving the following formula of the construction of the strophes: $\mathrm{a}+\mathrm{a}+\mathrm{ba}$. It is in the music, and not in the classical dimeter that provided the underlying structure, that we will find the explanation for this particular position on the third verse in the strophe». 


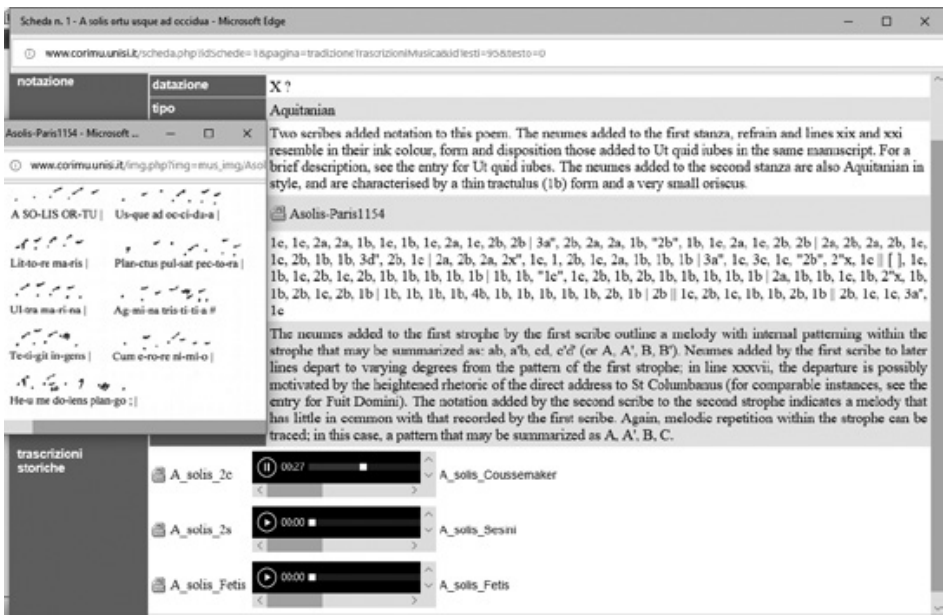

FIGURA 4. Scheda della notazione musicale presente nel manoscritto della BNF, lat 1154, testimone di $A$ solis ortu usque ad occidua, con aperta la finestra contenente la trascrizione neumatica (è possibile confrontare anche le trascrizioni storiche, moderne, e la relativa esecuzione).

I rhythmi sono, nella maggior parte dei casi, composizioni nate per essere cantate, più che lette, che rimangono di intento popolare, in quanto rivolte alla comunità dei fedeli, anche quando l'autore è tra i protagonisti della rinascita carolingia, nella quale convissero versi ritmici formalmente perfetti, come quelli di Paolino d'Aquileia, con quelli di Alcuino, che, a dispetto della rigorosa sequela del principio quantitativo nelle sue poesie metriche, compone rhythmi paradossalmente sfuggenti a qualsiasi tentativo di razionalizzazione nel computo delle sillabe; sono ritmiche, inoltre, parte delle raffinate opere di Gotescalco, le cui precoci costruzioni rimiche trovano confronti solo nella successiva poesia in lingua romanza.

Sono importanti anche le esclusioni operate dagli editori del Corpus, perché non tutto ciò che è cantato è poesia: si è delineata come una scala che va dalla prosa musicata, passa da quei versi che, pur partendo da modelli classici, hanno la melodia come elemento strutturante e presentano forti asimmetrie strutturali, fino ad arrivare ai rhythmi più complessi e regolari dal punto di vista stichico e ritmico, dove l'elemento melodico non è più necessario per comprendere la struttura dei medesimi.

Abbiamo, ad esempio, escluso dalla pubblicazione nel Corpus testi come Primus embolismus ${ }^{24}$ :

\footnotetext{
${ }^{24} \mathrm{Cfr}$. il testo in scriptio continua e neumato nel ms. BAV Vat. Lat. 3123, f. 46r. Link: <http://digi. vatlib.it/view/MSS_Vat.lat.3123> [consultato in data: 06/11/2019].
} 
Primus embolismus quarta nonarum decembris inseritur in secundo anno decennovenalis cycli.

Secundus quarto nonarum septembris erit inserendus in quinto eius anno.

Tercius pridie nonarum martii interponitur octavo anno.

Quartus in pridie nonarum decembris intercalatur anno undecimo.

Quintus in quarto nonarum novembris subrogatur in tercio decimo anno.

Sextus in quarto nonarum augusti immittitur in medietate sexti decimi anni.

Septimus tercio nonarum marcii admittitur in nono decimo cycli decennovenalis anno.

Lo troviamo nei codici trascritto come prosa neumata - la scriptio continua anche per la poesia ritmica è però una prassi non infrequente - di solito sino al settimo embolismo. Qui è stato aggiunto un altro brano con notazione che comincia Exigit octavus annus; né il primo, né il secondo testo sono accostabili a qualche forma ritmica, financo irregolare: non si tratta dunque di rhythmus cum melodia, bensì di prosa accostabile ai detti e alle filastrocche sul calendario cantilenate ancora oggi, del tipo «Chi vuole buon mosto zappi la vigna d'Agosto e chi vuol l'uva grossa zappi la proda e scavi la fossa». Nell'indagine condotta da Paul Evans ${ }^{25}$, la presenza di versi nei tropari provenienti da Saint-Martial, dunque da una delle maggiori fucine dell'arte paraliturgica, risulta in realtà esigua rispetto ad una prosa magari assonanzata $\mathrm{e}$ talora corredata di accorgimenti ritmici in chiusura; tra i versi, inoltre, rimane cospicua la presenza della metrica classica, specie con l'esametro leonino. Dunque, non tutto ciò che era cantato era poesia in versi.

Anche tra i rhythmi veri e propri, può essere grande la differenza stilistica, linguistica e metrica; il primo volume e le prime immissioni di testi nel database del CRM, trattando di poesie cantate trasmesse da collezioni non liturgiche, e dunque provenienti da contesti e rivolte a uditori assai diversi tra loro, offrono una preziosa testimonianza di questa varietà: sono rhythmi le poesie 'confessionali' di Paolino d'Aquileia, i planctus per la morte di Carlo Magno, per il maestro e abate Ugone, ma anche per lo scempio provocato dalla guerra (battaglia di Fontenoy), la poesia lirica di Gotescalco, gli indovinelli, le più 'popolari' canzoni narrative o didattico-morali, e quelle di tema apocalittico.

Nel Corpus possiamo distinguere altresì alcune tendenze comuni, le caratteristiche peculiari ai rhythmi e alcuni filoni privilegiati:

Si ha l'impressione che la musica abbia selezionato in questa raccolta una sorta di laboratorio di tendenze, di temi, modi ed espressioni della poesia che si

${ }^{25}$ Paul Evans, The Early Repertory of Saint Martial de Limoges, Princeton, Princeton University Press, 1970. 
imporrà nei secoli successivi, distillandone una sequenza di perle che rappresentano spesso dei vertici della letteratura di quest'epoca e la cui esecuzione musicata fa immaginare diffusi e conosciuti ben oltre la cerchia dei litterati ${ }^{26}$.

\section{Esempi di ricerca - rima, verso, strofa e genere letterario}

Il Corpus dei rhythmi mediolatini ha restituito una molteplicità di tipologie e di prassi versificatorie con le quali queste opere furono composte e fruite nel corso del tempo. Le considerazioni tratte dalla tradizione manoscritta e dai principali circuiti di trasmissione dei testi, la ricostruzione dei contesti e l'analisi comparata della versificazione permettono inoltre di ricostruire, caso per caso, le fonti cui gli autori attinsero, con soluzioni stichiche e strofiche distinte a seconda del bacino di riferimento.

Parimenti, si possono rilevare con esso i cambiamenti di gusti nel trascorrere dei secoli: la poesia ritmica, all'inizio identificata tale essenzialmente perché non metrica, diventa via via sempre più regolare sia negli stichi $\mathrm{e}$ nelle strofe, sia nelle figure di suono. Così accadrà successivamente con la sequenza - genere fortunato nel mediolatino come nella poesia volgare - che alla sua comparsa, nel nono secolo, mostra una struttura ancora molto libera e il finale monoassonanzato o con monorima, ma poi acquisisce, nel corso del decimo, la sua caratteristica conformazione binaria, e, nella prima metà del secolo successivo, adotta la versificazione ritmica. Tra decimo e undicesimo secolo - specie nelle sequenze di Adamo di San Vittore - assistiamo ad una ulteriore 'ritmizzazione', cioè ad una regolarizzazione sillabo-tonica agente su tutto il verso e non solamente sulla cadenza finale: il risultato è un verso più rigido, che permette però maggiori giochi di alternanza e la composizione di nuove strofe eterometriche, grazie anche all'utilizzo spesso non esornativo della rima che assume via via un ruolo sempre maggiore nella conformazione sia del verso che della strofa.

L'eterogeneità non è soltanto tematica, ma investe anche la forma, le modalità divulgative e di ricezione; presentiamo e contrapponiamo in tal senso due rhythmi editi nel CRM: la raffinata poesia di Gotescalco Ut quid iubes, pusiole e Quique cupitis audire, anonimo, anticipatore del Ludus de Antichristo.

\footnotetext{
${ }^{26}$ Francesco Stella, «Ad cantandum carmina. Testo e musica nel Corpus di ritmi latini musicati», in La poesia tardoantica e medievale, a cura di C. Burini De Lorenzi e M. De Gaetano, Atti del IV Convegno internazionale di studi (Perugia, 15-17 novembre 2007) in onore di Antonino Isola per il suo $70^{\circ}$ genetliaco, Alessandria, Edizioni dell’Orso, 2010, pp. 337.
} 


\section{CORPUS RHYTHMORUM MUSICUM}

\section{Riportiamo le relative schede linguistiche in primis:}

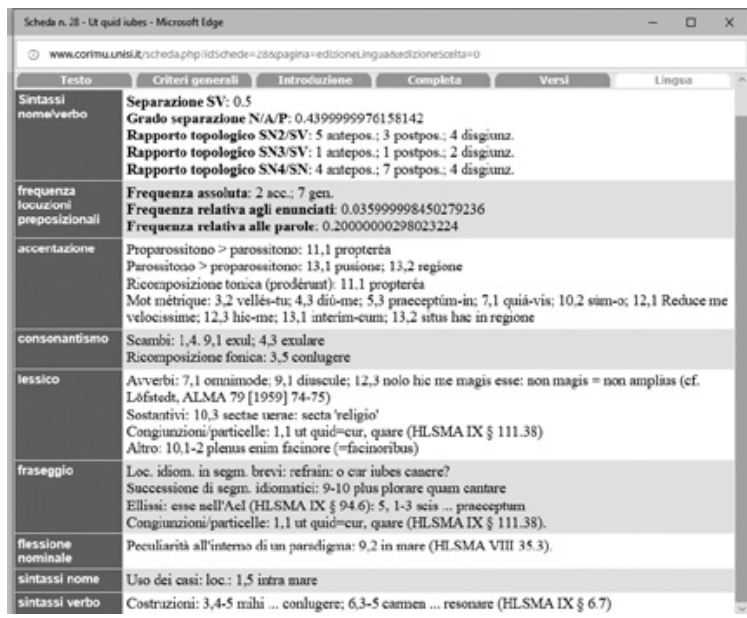

FIGURA 5. Scheda linguistica di Ut quid iubes (78 versi).

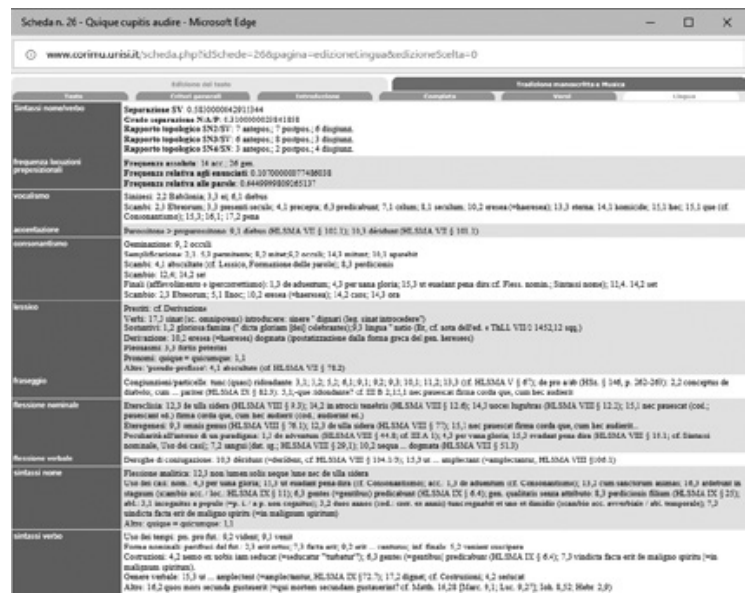

Figura 6. Scheda linguistica di Quique cupitis audire (54 versi).

\section{Legenda:}

$\mathrm{S} / \mathrm{V}$ : grado di separazione fra soggetto e verbo

N/A/P: grado di separazione tra nome e aggettivo

$\mathrm{SN} 2 / \mathrm{SV}$ : collocazione del complemento oggetto rispetto al verbo reggente

$\mathrm{SN} 3 / \mathrm{SV}$ : collocazione dei casi obliqui non retti da preposizione rispetto al verbo reggente SN4/SN: collocazione del genitivo rispetto al nome cui si riferisce

Frequenza assoluta: dei casi retti da proposizione, rispetto alle parole e ai verbi (enunciati) del componimento

HLSMA: Stotz, Peter, Handbuch zur lateinischen Sprache des Mittelalters, Voll. 2, München, Verlag C. H. Beck, 1996. 


\section{Irene VOLPI}

\section{Seguono le schede metriche:}

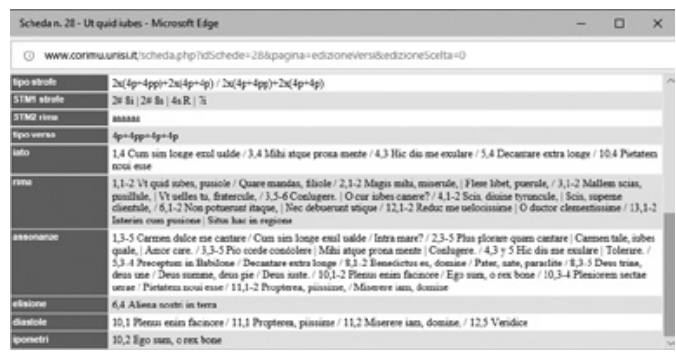

Figura 7. Scheda metrica di Ut quid iubes.

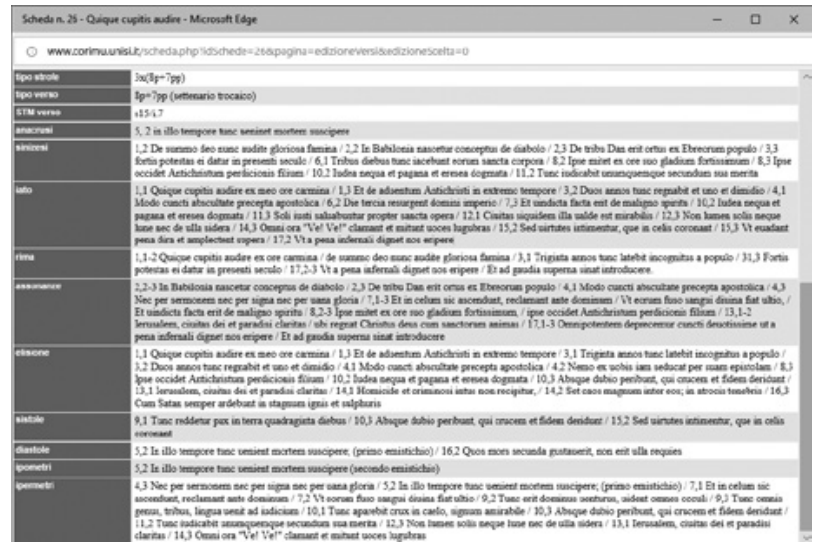

FIGURA 8. Scheda metrica di Quique cupitis audire.

Legenda

tipo verso ${ }^{27}$ :

numero $=$ quantità di sillabe

$\mathrm{p}=$ uscita parossitona

$\mathrm{pp}=$ uscita proparossitona

$\mathrm{x}+\mathrm{y}=$ verso con cesura

Es. $8 \mathrm{p}+7 \mathrm{pp}=$ verso formato da un ottosillabo parossitono e da un eptasillabo sdrucciolo= settenario (tetrametro) trocaico

stm verso ${ }^{28}$ :

numero $=$ quantità totale di sillabe

numero eventuale seguente $=$ quantità di sillabe dopo la cesura

$\mathrm{s}=$ uscita spondaica (piana)

$\mathrm{g}=$ uscita giambica (sdrucciola)

$/=$ uscita libera

$8 \mathrm{p}+7 \mathrm{pp}=\mathrm{s} 15 \mathrm{i} .7$

${ }^{27}$ Sistema di rappresentazione elaborato da Dag Norberg, cfr. An Introduction, op. cit.

${ }^{28}$ Sistema di codificazione progettato da E. d'Angelo per la codificazione informatica dei versi e delle strofe. Cfr. D’Angelo, Edoardo, «Sui sistemi di descrizione metrico-strutturale», in Poesia dell' Alto Medioevo europeo, op. cit., pp. 57-75; «STM -RL, Sistema Tassonomico Metricologico-Ritmi Latini (Terminologia, tassonomia, classificazioni della versificazione ritmica mediolatina)», in Poetry of the Early Medieval Europe, op. cit., pp. 75-104. 
Gotescalco, componendo versi brevi, con numerose cesure interne, rispetta anche con la sintassi l'andamento della versificazione; sono poche le locuzioni preposizionali, non insistito il ricorso a dieresi e diastole, mentre è frequente il ricorso al mot-metrique. Si registra un'unica ipometria al verso 10.2. L'autore è forse in carcere accusato di eresia e un giovane gli chiede di cantare un carmen dulce. Gotescalco risponde con una lamentatio che riprende motivi ovidiani, ma, mentre simula lo sconcerto per tale richiesta, anzi proprio da essa, fa scaturire il carme lirico agognato. Il lessico e il fraseggio sono dunque volutamente familiari: vedasi l'utilizzo insistito dei diminutivi, le proposizioni aderenti al parlato (fraseggio). La dolcezza e una ricercata immediatezza, oltre che dal lessico, sono dati dal verso e, soprattutto, dalla rima, già intesa in senso moderno, cioè coinvolgente le sillabe dall'ultima accentata e con funzione strutturale: una novità nella poesia mediolatina continentale che ha antecedenti solamente nella poesia delle Isole Britanniche ${ }^{29}$.

In Quique cupitis audire si contano quaranta casi di locuzioni preposizionali, a fronte dei nove di Gotescalco; sono presenti fenomeni di eteroclisia, deroghe di coniugazione, flessioni analitiche, uso dei casi e dei verbi anomalo e assai prossimo al volgare. Nella scheda metrica si ipotizza un caso di anacrusi, come accade non infrequentemente nei ritmi imitanti il tetrametro trocaico, specie quelli popolari del filone apocalittico individuato da Aval$1 \mathrm{e}^{30}$. La rima è occasionale ed è più che altro una estensione delle omofonie finali sino alla terzultima sillaba, più che una rima vera e propria, poiché la posizione dell'accento non è qui dirimente; frequentissima risulta l'elisione, generalmente evitata in ambito mediolatino (ma non in quello romanzo); sono presenti casi di sistole e diastole, ma soprattutto, si contano molti versi ipometri. Il controllo del verso e della sintassi è dunque minore, il pubblico è plurale e non dotto, l'intento morale e non lirico. La differenza con Ut quid iubes, anche nell'utilizzo della versificazione ritmica, è dunque profonda.

Il database del CRM può inoltre essere proficuamente interpellato per estrapolare dati relativi alle caratteristiche intrinseche ai generi e alla fortuna di determinate forme rispetto ad altre in specifici ambienti e periodi. Ci limitiamo qui a proporre un breve esempio nato da nostre precedenti ricerche: a fronte di molti esempi di trimetri giambici ritmici riscontrati nel CRM e nei

\footnotetext{
${ }^{29}$ Riguardo alla probabile derivazione della rima dell'Orbacense da modelli iberici - indagata tramite la tradizione manoscritta e la storia dei centri scrittori e culturali -, confronta Francesco Stella, "Gotescalco, la "scuola" di Reims e l'origine della rima mediolatina», in Il verso europeo. Atti del seminario di metrica comparata (4 maggio 1994), a cura di F. Stella, prefazione di C. Leonardi, Firenze, Consiglio Regionale della Toscana, Fondazione Ezio Franceschini, 1995, pp. 159-165.

${ }^{30}$ Cfr., ad esempio, «Preistoria dell'endecasillabo» di d'Arco Silvio Avalle in Le forme del canto, op. cit.
} 
Monumenta Germaniae Historica (MGH, PLAC IV) ${ }^{31}$, quali i Planctus per la morte di personaggi importanti o cari amici, nessun componimento con medesima versificazione compare, ad esempio, né nel B. N. lat. 1139 (proveniente dalla fucina di Saint Martial de Limoges e d'importanza capitale sia per le parti in volgare che per quelle latine, ormai ritmiche e saldamente inquadrate in strutture strofiche, specie nella prima sezione riservata all'Officio $^{32}$, né nella raccolta Cantabrigiense (che conserva un gran numero di sequenze extraliturgiche, utilizzate in ambito amoroso, narrativo - con scene simili al fabliau romanzo -, oppure di lode o il compianto per personaggi importanti).

\begin{tabular}{|c|c|}
\hline Versi e strofe & n. attestazioni \\
\hline $8 / 9+7 / 8$ & 4 \\
\hline $8 p+7 p p$ & 5 \\
\hline $2 x(8 p+7 p p)$ & 5 \\
\hline $\mathrm{R}$ & 7 \\
\hline $3 x(8 p+7 p p)$ & 27 \\
\hline $\mathrm{R}$ & 1 \\
\hline $4 x(8 p+7 p p)$ & 6 \\
\hline pseudosaffica & 1 \\
\hline
\end{tabular}

Versi e strofe
\begin{tabular}{|l|r|}
\hline esametro ritmico & attestazioni \\
\hline $6 p+8 p$ & 18 \\
\hline \multicolumn{2}{|c|}{$\mid r(13,4 \%)$} \\
\hline R $(8 p+4 p)$ & 1 \\
\hline $5 x(8 p+4 p)$ & 1 \\
\hline
\end{tabular}

\begin{tabular}{|l|r|}
\hline $2 x(5 p+6 p p)+R$ & 2 \\
\hline saffica & 1 \\
\hline
\end{tabular}

\begin{tabular}{|c|c|}
\hline \multirow{3}{*}{$\begin{array}{l}\frac{5 p+7 p p}{2 x(5 p+7 p p)} \\
R\end{array}$} & 1 \\
\hline & \\
\hline & 6 \\
\hline $3 x(5 p+7 p p)$ & 4 \\
\hline $\mathrm{R}$ & 1 \\
\hline $4 x(5 p+7 p p)$ & 1 \\
\hline $\mathrm{R}$ & 2 \\
\hline $5 x(5 p+7 p p)$ & 8 \\
\hline pseudosaffica & 4 \\
\hline
\end{tabular}

\begin{tabular}{|l|r|}
\hline $4 p+7 p p$ & 2 \\
\hline $9 p p$ & 1 \\
\hline $8 p$ & 5 \\
\hline \multicolumn{2}{|l|}{} \\
\hline $7 p$ & 3 \\
\hline solo nel Rit. & 2 \\
\hline
\end{tabular}

\begin{tabular}{|l|l|}
\hline $7 \mathrm{pp}$ solo nel Rit. & 1 \\
\hline
\end{tabular}

${ }^{31}$ Il volume raccoglie la poesia latina merovingia e carolina (VI-IX sec). Monumenta Germaniae Historica, Monumenta Germaniae Historiae und Bayerischer Staatsbibliothek / Digitale Bibliothek, 2010. Link: <http://www.mgh.de/> [consultato in data: 06/11/2019]; Monumenta Germaniae Historica - eMGH, Brepolis, $<$ http://asbproxy.unisi.it:3222/eMGH/pages/Search.aspx > [consultato in data: 06/11/2019].

${ }^{32}$ Contiene i tropi Be deu hoi mais finir nostra razos, O Maria deu maire e $i$ bilingui Sponsus e In hoc anni circulo. 


\begin{tabular}{|l|r|}
\hline $8 p p+8 p p$ & 1 \\
\hline $4 x(8 p p)$ & 6 \\
\hline$R$ & 3 \\
\hline $6 x(8 p p)$ & 1 \\
\hline
\end{tabular}

$\mathrm{R}=$ Forme ritornellate

\begin{tabular}{|l|r|}
\hline $2 x(6 p p+6 p p)+R$ & 1 \\
\hline $6 p p$ & 1 \\
\hline $6 p$ & 1 \\
\hline $5 / 6$ & 1 \\
\hline $5 p$ & 4 \\
\hline
\end{tabular}

FIGURA 9. Numero di attestazioni delle tipologie stichiche e strofiche in CRM e MGH

Ciò presuppone o che già in ambito mediolatino il verso $5 p+7 p p$ abbia subito trasformazioni notevoli, quali la non obbligatorietà della cesura fissa dopo la quinta sillaba, oppure che, almeno, non sia stato accolto tra i ritmi ammessi nelle sequenze (risulta altresì escluso dalle sperimentazioni ritmiche di Adamo da San Vittore) e che in questo ambito ne sia decaduto l'uso.

Tra i rhythmi popolari, invece, si individua un gruppo contiguo alla liturgia - rappresentato primariamente dal filone penitenziale-escatologico - in cui è presente una componente anisosillabica che non intacca generalmente l'uscita finale, rispettata per il mantenimento del verso ritmico, ma che può indurre a fenomeni di anacrusi. La forma più utilizzata in questo caso è il settenario trocaico, che poi subisce numerose evoluzioni e variazioni nei secoli successivi, quali la separazione dei due emistichi, la reiterazione di uno di essi, l'inserzione di diversi giochi di rime; come esempio ben conosciuto e fortunato si può citare la sequenza Stabat mater dolorosa, le cui strofe sono formate da due ottosillabi piani (a loro volta suddivisibili in $4 p+4 p$ ) tra loro in rima, e un eptasillabo sdrucciolo finale rimante con quello successivo (aab ccb dde ffe, etc.).

In sintesi, il trimetro giambico, in uso nel periodo carolingio in ambito più ristretto e formalizzato, tende a scomparire; il settenario trocaico, utilizzato in generi popolari e poi sfruttato anche per i componimenti paraliturgici, ha una enorme fortuna anche nei secoli successivi (si pensi che quando Manzoni scriverà i suoi Inni Sacri, per attenersi alla tradizione, comporrà l'inno per la Resurrezione con ottonari piani chiusi da uno sdrucciolo, corrispondenti alla forma latina del settenario trocaico ritmico: È risorto: or come a morte / la sua preda fu ritolta? / Come ha vinto l'atre porte, / come è salvo un'altra volta / quei che giacque in forza altrui? / io lo giuro per Colui / Che da' morti il suscito $\left.{ }^{33}\right)$.

${ }^{33}$ Biblioteca della Letteratura Italiana, link: $<\mathrm{http}: / /$ www.letteraturaitaliana.net/pdf/Volume_8/t338. pdf $>$ [consultato in data: 06/11/2019], p. 9; dall'edizione: Alessandro Manzoni, Opere, a cura di R. Bacchelli, Milano-Napoli, Ricciardi, 1973. 


\section{Work in progress - conclusioni provvisorie}

Come si vede, la prima immissione dei rhythmi musicati dal iv al ix secolo in manoscritti non liturgici, ha aperto all'opportunità di un'evoluzione nella ricerca in campo metrico, linguistico e filologico (attributivo), nonché riguardo agli studi sui generi letterari, condotti, in modo particolare, nei due convegni $\mathrm{i}^{34}$ preparatorî all'edizione del primo volume e alla creazione del database del Corpus Rhythmorum Musicum. I contributi sono continuati per la seconda immissione riguardante i rhythmi computistici - relativi all'insegnamento e alla memorizzazione di questioni astronomiche, zodiacali e del calcolo della Pasqua - con la scoperta di nuovi manoscritti testimoni, gli studi relativi alla produzione di Pacifico da Verona ${ }^{35}$ e tesi su versi computistici metrici. Segnaliamo, soprattutto, l'edizione curata da Chiara Savini e ora nel CRM, del ritmo Anni Domini notantur, per la quale sono stati scoperti e collazionati 9 manoscritti nuovi rispetto a quelli considerati nell'ultima edizione del rhythmus: essa, dunque, getta luce sull'ingente opera di ricognizione e di ricerca ancora da mettere in atto su questa tipologia di componimenti, pur tenendo conto della monumentale opera di Borst al riguardo ${ }^{36}$, e sul rinnovamento degli studi nel settore a partire dal lavoro sorto per e grazie al CRM.

Con la terza sezione del Corpus verranno aggiunti gli inni con versificazione ritmica, prodotti entro il IX secolo e con una o più melodie connesse, che siano attestate in codici redatti entro il Duecento. Ci pare una scelta che può essere fruttuosa per più motivi. In primis, l'antichità delle attestazioni degli inni di fattura ritmica (alcuni di essi, come il Christe qui lux es et dies, sono citati già nelle Regulae di Cesario e Aureliano di Arles) dà l'opportunità di indagare questo tipo di versificazione anche nei secoli più alti del medioevo; in secondo luogo la chiara derivazione di questi versi dai loro omonimi quantitativi, in special modo dai fortunatissimi dimetri giambici ambrosiani,

\footnotetext{
${ }^{34}$ Poesia dell'alto medioevo europeo, op. cit. e Poetry of the Early Medieval Europe, op. cit. Ma cfr. anche Francesco Stella, «Ad cantandum carmina», op. cit.; «Indicatori di prossimità al protoromanzo: applicazioni sperimentali alla poesia ritmica altomedioevale», in Latin vulgaire - latin tardif, a cura di C. A. Abellán, Sevilla, Universidad de Sevilla, Secretariado de Publicaciones, 2006, pp. 549-563; Francesco Stella, «Appunti per una fenomenologia linguistica della forma canzone dal medioevo a De André», in Semicerchio, 44 (2011), Da Carlo Martello a Il nome della rosa, pp. 9-21. Ora in versione aggiornata in inglese per gli Atti della Atkinson Conference di Columbus (Ohio) 2011, a cura di G. Boone; «Les premières définitions de "rythme" en latin médiéval et les poèmes sur les rythmes du temps», in Rythmes et croyances au Moyen Age. Actes de la journée d'étude organisée par le Groupe d'anthropologie historique de l'Occident médiéval (Centre de recherches historiques, EHESS/CNRS), a cura di M. Formarier e J. Schmitt, Paris, Institut National d'Histoire de l'Art (INHA), (Scripta mediaevalia 25), 2014, pp. 27-45.

${ }^{35} \mathrm{Cfr}$. «Les premières définitions de "rythme"», op. cit.

${ }^{36}$ Arno Borst, Der Streit um den karolingischen Kalender, Hannover, Hahnsche Buchhandlung, 2004; Schriften zur Komputistik im Frankenreich von 721 bis 818, Hannover, Hahnsche Buchhandlung, 2006.
} 
può favorire un'analisi dei comportamenti metrici molto dettagliata; l'utilizzo diffusissimo e la contiguità con altri generi 'nuovi' della paraliturgia come i tropi e le sequenze, inoltre, può aiutare a far luce su alcune evoluzioni interne alla ritmica mediolatina, nonché sugli scambi tra questa e la nascente poesia in volgare. Infine, un elemento fondamentale sia per la comprensione di questi testi, sia per l'impostazione storica e letteraria del Corpus, è il rapporto con il contesto liturgico per il quale vennero creati o rimaneggiati: come per l'elemento musicale, anche questo aspetto non può essere considerato esterno e avulso dall'analisi filologica e letteraria. Le fonti di questi testi sono correlate alla loro funzione liturgica e agli altri brani utilizzati in quella occasione, oltre che ad altri inni con funzione simile: un testo dedicato a un santo, trarrà informazioni e intere locuzioni dagli Acta, dalle collette, antifone, responsori dedicati alla sua commemorazione, ma anche da altri inni dedicati alla stessa tipologia di beatus (locale, d'epoca paleocristiana, recente, martire, confessore, vergine, etc.). Quando all'inno viene cambiato il contesto liturgico (accadimento tutt'altro che infrequente), $o$, più semplicemente, nel passaggio da un monastero (o una chiesa) all'altro, spesso viene sottoposto a innovazioni sostanziali, tanto da dover parlare di più versioni dello stesso testo: talvolta si aggiungono elementi narrativi, oppure ci si limita a cambiare la dossologia, talora invece si interviene su tutto il brano e lo si semplifica, nel lessico e nella sintassi, per renderlo più comprensibile all'uditorio incolto. In altri casi i testi riportati nei codici fanno presupporre una trasmissione non scritta, ma orale, anzi diremmo di esecuzione musicale, in cui le singole parole vengono travisate, ma non l'elemento ritmico-fonico, mantenuto nella memoria dell'emanuense grazie alla melodia. La pluralità di trascrizioni melodiche tramandate per un singolo inno, dunque, non esclude la possibilità di estrapolare informazioni importanti nel raffronto del testo poetico con quello musicale.

Gli esempi e i problemi che abbiamo presentato in questa sede non esauriscono le possibilità di ricerca e sintesi dei dati possibili grazie al Corpus; qui ci è piaciuto soltanto suggerire alcune tematiche ancora aperte: il rapporto del testo con la musica e quanto questa definisca la forma della poesia, la vicinanza o meno dei componimenti ritmici a quelli che possono essere considerati i due poli opposti, vale a dire la poesia tardoantica (più che classica) e quella romanza (e quale tipo di poesia in volgare), e dunque la rilevazione di un eteromorfismo ingente e su più livelli (stichico, strofico, linguistico, di occasione e di pubblico, di modelli e di fortuna) all'interno del gruppo dei rhythmi. 


\section{Irene VOLPI}

Il CRM non è perciò stato concepito solamente come una trasposizione di dati in formato elettronico ${ }^{37}$, ma come corpo vivo, in crescita, che ha lasciato, e speriamo continui a lasciare, tracce di sé nel campo della ricerca.

Inviato: $10 / 12 / 2018$

Accettato: 29/10/2019

${ }^{37}$ Contenuto nota: Entro febbraio 2020 verrà aggiornato il front-end del sito per migliorarne la visualizzazione e facilitare la ricerca. 


\section{Il CORPUS RHYTHMORUM MUSICUM}

\section{OPPORTUNITÀ E PROSPETTIVE DI UNA EDIZIONE CRITICA DIGITALE}

Riassunto: Il Corpus Rhythmorm Musicum (CRM) è un progetto ideato da Francesco Stella, che dà edizione critica dei rhytmhi - poesie latine medievali basate su un principio sillabico e accentuativo - che siano prodotti dal quarto al nono secolo e provvisti di almeno una melodia attestata nella tradizione manoscritta. A seguito della pubblicazione del primo volume e del CD-ROM (Firenze, SISMEL, Edizioni Del Galluzzo, 2007), contenenti canti di tradizione non liturgica, Luigi Tessarolo ha riprogrammato il database per il web nel 2011. Nel database, liberamente accessibile online $(<\mathrm{http}: / / \mathrm{www} . c o r i m u . u n i s i . i t />)$, per ogni rhythmus, oltre all'edizione digitale di poesie e musiche (curate da $\mathrm{S}$. Barrett), è possibile visualizzare: tutti i manoscritti collazionati e le loro descrizioni; le trascrizioni di poesie e melodie nei diversi testimoni (insieme a quelle alfanumeriche e alle esecuzioni musicali dirette da G. Baroffio, curatore del reperimento delle trascrizioni moderne); le schede con il profilo storico, letterario ed ecdotico, quelle su versi e lingua. Da esse derivano le tavole linguistiche di C. Cenni, F. Sivo, P. Stoppacci e le statistiche lessicali, un repertorio delle forme ritmiche curato da E. D’Angelo e C. Pérez González e un menù di ricerca che permette di porre domande complesse al database. Si presentano in questa sede le possibilità d'analisi offerte dal CRM e si indaga sul ruolo svolto dall'elemento melodico nella formazione di questi componimenti. S'indicano, infine, alcuni studi sorti grazie al database, nonché i progetti in corso: l'edizione dei rhythmi computistici - su calendario, astronomia e metodo per calcolare la data della Pasqua - e gli inni ritmici.

PARole ChIAVE: Rhythmus, Alto Medioevo, Edizione digitale, Manoscritti, Melodia.

\section{The CORPUS RHYTHMORUM MUSICUM}

\section{OPPORTUNITIES AND PERSPECTIVES OF A DIGITAL CRITICAL EDITION}

ABSTRACT: The Corpus Rhythmorm Musicum (CRM) is a project created by Francesco Stella; here there are the critic editions of rhytmhi - Medieval Latin poems based on syllables and stretches - written from iv to ix century and provided at least with one melody transmitted by the manuscript tradition. After the first volume and CD-ROM publication (Florence, SISMEL, 2007), containing songs from non-liturgical sources, Luigi Tessarolo has reprogrammed the database for the web on 2011. In the database, freely accessible online ( $<\mathrm{http}$ ://www.corimu.unisi.it/ $>)$, for every rhythmus, in addition to the digital edition of poems and music (edited by S. Barrett), you can view all the collated manuscripts and their descriptions; the transcriptions of poems and melodies (together with the alphanumeric ones and the musical per- 
formances directed by G. Baroffio, who also found the modern transcriptions); the introductions with historical, literary and ecdotic profiles, the tabs Versi and Lingua. From these originate the linguistic tables edited by C. Cenni, F. Sivo, P. Stoppacci, the lexical statistics, a repertoire of rhythmic forms edited by E. D'Angelo and C. Pérez González and a search menu that allows you to ask complex questions to the database. Here we present the analysis possibilities offered by the CRM and we investigate the role played by the melodic element in the formation of these compositions. Finally, we indicate some studies arisen thanks to the database, as well as the ongoing projects: the edition of the rhythmi computistici - on calendar, astronomy and method for calculating Easter - and the rhythmic hymns.

KeYwords: Rhythmus, High Middle Age, Digital Edition, Manuscripts, Melody. 